\title{
Does Drug-drug Interactions Attain Enough Attention from Health Care Providers?
}

\section{Hamoudi NM*}

Department of Pharmaceutical Chemistry, Gulf Medical University, Ajman, United Arab Emirates

\begin{abstract}
Every year many patients are affected by adverse drug events that result from the complication of drug therapy. Drug-Drug Interactions (DDIs) are an expected subtype of adverse drug events. Most potential DDIs are preventable, but it remains a significant problem to patients and the health care system. Among the risk factors associated to DDls include older age, comorbidities, polypharmacy and hospital stay for long time. DDIs are a considerable clinical problem in the hospitalized cardiac patients, cancer patients, hypertensive patients, and patients with immunodeficiency virus (HIV) and hepatitis C virus (HCV) infections. Best management of DDIs involves the assessment of the interaction; decision to prescribe, dispense, or manage the interacting combination; follow-up monitoring and suitable patient counseling. Different studies were performed to detect and avoid DDIs. Different strategies have been introduced to reduce and prevent the risk of DDIs through the improvement of labeling on metabolic profile for new drugs and serious drug-drug and drug -gene combination, determination the effect of new guideline on product labeling changes for elderly patient and patients with polypharmacy. In clinical practice pharmacist can use good information sources; clinicians can use screening software for the detection and management of DDIs; consumers and patients can use decision support systems for potential DDIs and medication reconciliation should be considered by healthcare professionals to reduce medication error.
\end{abstract}

Keywords: Drug-drug interactions; Risk factors; Polypharmacy; Preventive strategies

\section{Introduction}

The main challenges in clinical practice involve drug related problems such as adverse drug reactions, Drug-Drug Interactions (DDIs), idiosyncratic reactions, and hypersensitivity reactions [1]. Adverse drug events are one of the important complications of medication therapy which could affect million patients every year. They can lead to serious consequences and elevate healthcare expense [2]. Drug-drug Interactions (DDIs) are an expected subtype of adverse drug events. DDIs are a significant clinical and public health problem particularly in old age people. This problem becomes more burden as people age and polypharmacy increases. Although harmful interactions has been known but the incidence of adverse drug events and hospitalization rate associated to DDIs is still high. This may be due to either insufficient information about drug history of patients or inadequate knowledge of prescriber [3]. The prevention of most adverse drug events can be achieved by identifying potential DDIs [4], which are varied from minor to severe events that may be lethal [5]. Studies concerning the causes of people admitted to hospital shows that up to $27.0 \%$ of the patients have complications that are due to the consequences of DDIs [6].

\section{Mechanism of Drug-drug Interaction and Therapeutic Drug Classes}

DDIs can be classified to pharmacokinetic or pharmacodynamics interactions. Pharmacokinetic drug interactions are happened when one drug increase or interfere with absorption, distribution, metabolism, or excretion of another drug and lead to a change in drug concentration in the body $[7,8]$. Drug metabolism is the most important pharmacokinetic drug interaction because the detoxification of most drugs occurs in the liver and this can be affected by genetics, which explains why some patients experience adverse effect from some drug combinations while others do not. It was observed that the potential DDIs to be one of the challenges that may cause a change in pharmacokinetic and pharmacodynamics of drugs then change the gross therapeutic response $[5,9]$. The difference between potential and actual DDIs was stated by consensus panels [10]. In case of potential DDI two drugs known to interact are prescribed at the same time although adverse incidence occurs. While actual DDI is the change in clinical effect of target drug due to concurrent administration of another drug ((precipitant drug).

A study identified that CYP3A contribute for $2 / 3$ of all interactions. The antivirals and oncology drugs represent the most therapeutic drug classes with significant risk of DDIs [11]. Drugs used for the treatment of human immunodeficiency virus (HIV) and hepatitis $\mathrm{C}$ virus (HCV) infections represent a specific challenge regarding the management of actual and potential DDIs. The mechanism of these DDIs involves inhibition or induction of activity of human cytochromesP450, energydependent transport proteins and glucuronosyl transferases. DDIs can be complex and time dependent in nature. The potential DDIs cannot be all evaluated by clinical pharmacokinetic studies in the case of the progression of HIV, HCV, and substance abuse treatments because time and resources available for the development of new drug are limited. There is a need for further strategies to revise screening techniques and in vitro models to promote more efficient targeting methods for these clinical studies with the highest effect to increase medication effectiveness and patient safety [12]. It is very important

*Corresponding author: Nehad M Hamoudi, Department of Pharmaceutical Chemistry, Gulf Medical University, Ajman, United Arab Emirates, Tel: +1-519-6975783; E-mail: nehadmh@gmail.com

Received October 11, 2018; Accepted November 13, 2018; Published November 20, 2018

Citation: Hamoudi NM (2018) Does Drug-drug Interactions Attain Enough Attention from Health Care Providers? Pharm Anal Acta 9: 598, doi: 10.4172/21532435.1000598

Copyright: $\odot 2018$ Hamoudi NM. This is an open-access article distributed unde the terms of the Creative Commons Attribution License, which permits unrestricted use, distribution, and reproduction in any medium, provided the original author and source are credited. 
to determine DDIs for anticancer agents because they have narrow therapeutic index and potential toxicity. Recent study reported that most of DDIs in ambulatory patients with cancer were classified as pharmacokinetic [13] in contrary to previous studies that reported anticancer agent interaction with other drugs can result in some changes in its pharmacokinetics or pharmacodynamics activity with significant change in toxicity or efficacy [14]. This difference may result from the different tumor types and specific concomitant treatment as well as the database used for the assessment of interaction [13].

\section{DDIs Associated Risk Factors and Possible DDIs in Different Diseases}

Among the risk factors associated with DDIs is older age (likely because of more comorbid illnesses among the elderly). Many studies noticed that elderly people are more susceptible for the exposure to chronic cases; they usually have multiple diseases as well as multiple medications [15]. Another study reported that $26.5 \%$ of elder people taking medications were exposed to at least one DDI [16]. However polypharmacy and hospital stay for long time have an impact on the occurrence rate of potential DDIs [15]. It was reported that $75.9 \%$ of patients taking seven or more drugs were exposed at least to one potential DDI. However $73.8 \%$ of patients reside for seven days in the hospital or more were at the risk of DDIs [17]. The potential drug interactions were identified in $31 \%$ of cancer patients receiving supportive care exclusively [18]. A recent prospective study with ambulatory cancer patients also found clinically relevant DDI in $27 \%$ of cases [19]. Medication classes that interact with chemotherapy are: antacids, antibiotics, anticoagulants, antiemetic, anticonvulsants, antifungal agents, anti-retroviral agents, corticosteroids and NSAIDs. A prospective conducted in ambulatory patients with cancer treated with oral and/or intravenous drugs [13] (mechanism of interaction /recommended intervention to overcome such interactions): Acetaminophen-Imatinib (imatinib inhibits O-glucuronidase /stop acetaminophen); Carbamazepine-Paclitaxel (carbamazepine induces CYP2C8 and may increase the metabolism of paclitaxel/consider carbamazepine replacement); Fluoxetine- Tamoxifen (fluoxetine inhibits CYP2D6 and may reduce the therapeutic effect of tamoxifen/ stop fluoxetine); Hydrochlorothiazide-paclitaxel (this combination may increase the risk for hypotension/consider diuretic replacement); Ibuprofen- Methotrexate (ibuprofen impairs renal excretion of methotrexate/stop ibuprofen); Irbesartan-Tamoxifen (irbesartan inhibits CYP2C9 and may reduce the metabolism of tamoxifen /monitor adverse effects of tamoxifen); Loratadine-Tamoxifen (tamoxifen inhibits P-glycoprotein/ABCB1 and may increase loratadine adverse effects/monitor therapy); Omeprazole-Erlotinib (omeprazol may reduce GI absorption of erlotinib/replace proton pump inhibitor); Omeprazole-Tamoxifen (omeprazole inhibits CYP2C9 and may reduce the metabolism of tamoxifen/monitor electrocardiogram); VerapamilPaclitaxel (paclitaxel inhibits CYP3A4 and may increase verapamil concentration/consider verapamil replacement and Monitor blood pressure); Warfarin- Sorafenib (sorafenib inhibits CYP2C9 and may reduce the metabolism of warfarin/use LMWH instead of warfarin and monitor for bleeding and labile INR values) [13].

DDI is a considerable clinical problem in the hospitalized cardiac patients $[20,21]$. Studies revealed that cardiovascular patients are more often exposed to potential DDIs as compared to patients with other diseases [22,23].The reason behind this is due to old age, polypharmacy, and pharmacokinetic or pharmacodynamics nature of drugs used in cardiology [24]. Data base development in hospitals may help for the control of potential DDIs in hospitalized cardiac patients [25]. These studies show that potential DDIs are one of most important issues in cardiac patients. A cross-sectional descriptive study carried at the department of cardiology report the combination of drugs that result in DDIs/ severity of interaction and (scientific evidence of the potential DDIs classified as: Established show the presence of interaction by researchers; Theoretical show the presence of interaction but there is no proof or controlled studies; Probable suspect presence of interaction but there is no evidence): Asprin- Clopidogrel/major severity (Probable interaction increase bleeding); Clopidogrel - Fondaparinux/major severity (Theoretical interaction); Aspirin-Fondaparinux/major severity (Theoretical interaction); Aspirin- Bisoprolol/moderate severity (Probable interaction); Asprin-Ramipril /moderate (Established); Aspirin-Nitroglycerin/moderate (Probable); HydrochlorothiazideRamipril/moderate (Probable); Aspirin-Lisinopril moderate (Established); Atorvastatin-Clopidogrel/moderate (Established) [24].

Patients with hypertension are particularly exposed to DDIs due to the presence of factors associated with DDIs such as old age, polypharmacy and comorbidities [26]. A prospective, cross-sectional study was conducted among the hypertensive patients in medicine department [26] finds the common interaction among antihypertensive combination: Atenolol- Amlodipine (increased risk of hypotension), Furosemide- Enalapril (acute hypotension and increased risk of renal insufficiency), Furosemide-Telmisartan (telmisartan increases serum $\mathrm{K}$ level and furosemide decreases serum Klevel), and Furosemide-Atenolol (affects serum $\mathrm{K}$ level). The combination of Atenolol and Amlodipine has opposite effect on plasma renin activity, and double mechanism can be beneficial in controlling hypertension. Also they found that these combinations: Spironolactone-Enalapril, Spironolactone-Telmisartan, and Spironolactone-Carvedilol also had caused potential DDIs with an increase in serum potassium levels. Among the antihypertensive drugs interacting with other cardiovascular drugs it was reported that the combination of Digoxin-Spironolactone, Digoxin-Enalapril, and Digoxin-Furosemide was the potential drug pairs causing drug interactions. Spironolactone could increase Digoxin concentration by decreasing its clearance [27]. There is a recurrent DDI between the antihypertensive drugs and NSAIDs, such as Enalapril-Diclofenac. Diclofenac is a cyclooxygenase inhibitor, decreases the action of enalapril by inhibiting the prostaglandin mediated vasodilating effect of ACE inhibitors and also increased the risk of renal function deterioration by compromising renal hemodynamics [28].

\section{Strategies for Reducing and Preventing the Risk of Drug Interactions}

Among the strategies involved to decrease and prevent the risk of DDIs, regulatory effort is required to improve labeling on metabolic profile for new drugs and serious drug-drug and drug -gene combination [29]; Pharmacogenetics is part of clinical pharmacology that help to predict patient's metabolizer phenotype and aid the choice of therapy, pharmacogenetics is used now in many countries; and the application of quality index like population surveillance for clinically significant drug interactions is recommended [29]. Drug labeling is a complex issue that requires balance between clinical relevance, consistency of information and substantive data quality. There is need to determine the effect of new guideline on product labeling changes for elderly patient and patients with polypharmacy [30].

The DDI is more recurrent threat in oncology due to the exponential development in the number of modern treatment choices. The awareness about the potential DDIs in patients that generally are exposed to polypharmacy and the development of institutional planning to reduce the hidden dangers are important to avoid damage to patient's health. Further studies should concentrate on the identification of the actual DDI and improvement of the protection strategies to reduce the risk of 
DDI [31]. Interactions with other medications can cause small changes in the pharmacokinetics or pharmacodynamics of a chemotherapy agent that could significantly alter its efficacy or toxicity. Improvements of in vitro methods and early clinical testing can help in the prediction of potential and clinical significant DDIs [32].

Some drugs don't have adverse clinical effect. However recognizing every DDI is impractical. Pharmacist can use these two good sources of information to prevent and control any further DDIs: Drug Interaction Facts [33] and Drug Interactions Analysis and Management: A Clinical Perspective and Analysis of Current Developments [34]. In clinical practice, drug alert software programs have been developed. Clinicians can use screening software for the detection and management of DDIs. For the detection of clinically important DDIs program a comparison between five common DDI programs were performed and they found that Lexi-Interact and Micromedex were the best in performances. However more accurate result can be achieved by the combination of more than one program with the intervention of expert pharmacist [35].

One possible solution that has been proposed is to use a decision support system to detect and avoid potential DDIs [36,37]. With the rise of smartphones and mobile apps, decision support systems for potential DDIs are now within the reach of consumers and patients. Recent study searched the most common mobile app databases and found 23 apps that can check for potential DDIs. However some of these apps provided good quality, accurate, and comprehensive information about potential DDIs while other apps does not conformed to high standards. This study also identified secondary features such as medication reminder, refill reminder, medication history tracking, and pill identification could help enhance the effectiveness of potential DDI apps [38]. A study found that utilizing clinical decision support software in older adults with cancer was beneficial for detecting DDIs however, variability existed with severity reporting. Future studies need to identify the relevant DDIs with clinical implications in order to optimize medication safety in this population [39].

Recent study reports the presence of methods to reduce DDIs warnings which are possibly to be overridden exist across medication, supplier and patient encounters. But it seems that more strong and clever tools are required. There are specific features present at the hospital, medication, supplier and patient level which can be used to assist specialize and reinforce information transition.

Many studies were report the importance of medication reconciliation to avoid DDIs. $46 \%$ of medication error and $20 \%$ of adverse drug reactions occur in the hospital setting due to the lack of medication reconciliation [40]. Therefore medication reconciliation should be considered to reduce medication error as well as many efforts should be performed to foster all healthcare professionals to implement a medication reconciliation process [41].

\section{Conclusion}

DDIs and adverse drug events represent serious problem and must be considered by all healthcare professionals. DDIs is widely expected in patients with cancer, cardiovascular, hypertension and immunodeficiency virus (HIV) and hepatitis $\mathrm{C}$ virus (HCV) infections due to the presence of DDIs associated risk factors. Potential DDIs cannot be all evaluated by clinical pharmacokinetic studies in the case of the progression of HIV, HCV, and substance abuse treatments due to the limitation of time and resources available for the development of new drug. There is a need for further strategies to revise screening techniques and in vitro models to promote more efficient targeting methods for these clinical studies with the highest effect to increase medication effectiveness and patient safety. Potential DDI should be identified first in order to determine whether the patient at risk of harmful DDIs. Different strategies to prevent and reduce the risk of DDIs have been introduced. Improvement of labeling on metabolic profile for new drugs and serious drug-drug and drug -gene combination is required, determination the effect of new guideline on product labeling changes for elderly patient and patients with polypharmacy and the improvements in vitro methods and early clinical testing can help in the prediction of potentially clinically significant drug interactions. In clinical practice, pharmacist can use good information source; clinicians can use screening software for the detection and management of DDIs; consumers, patients can use decision support systems for potential DDIs; and healthcare professionals should consider medication reconciliation to reduce medication error.

\section{References}

1. Krähenbühl-Melcher $A$, Schlienger R, Lampert $M$, Haschke $M$, Drewe J, et al (2007) Drug-related problems in hospitals. Drug Saf 30: 379-407.

2. Becker ML, Kallewaard M, Caspers PW, Visser LE, Leufkens HG, et al. (2007) Hospitalisations and emergency department visits due to drug-drug interactions: a literature review. Pharmacoepidemiol Drug Saf 16: 641-651.

3. Askari M, Eslami S, Louws M, Dongelmans D, Wierenga P, et al. (2012) Relevance of drug-drug interaction in the ICU-perceptions of intensivists and pharmacists. Studies Health Tech Inform 180: 716-720.

4. Hansten PD, Horn JR (2006) Drug interactions: Analysis and management Wolters Kluwer Health, USA

5. Baxter K, Preston CL (2010) Stockley's drug interactions. Pharmaceutical Press, London.

6. Janchawee B, Wongpoowarak W, Owatranporn T, Chongsuvivatwong V (2005) Pharmacoepidemiologic study of potential drug interactions in outpatients of a university hospital in Thailand. J Clin Pharm Ther 30: 13-20.

7. Riechelmann RP, Zimmermann C, Chin SN, Wang L, O'Carroll A, et al. (2008) Potential drug interactions in cancer patients receiving supportive care exclusively. J Pain Symptom Manag 35: 535-543.

8. van Leeuwen RW, Jansman FG, van den Bemt PM, de Man F, Piran F, et al. (2015) Drug-drug interactions in patients treated for cancer: A prospective study on clinical interventions. Ann Oncol 26: 992-997.

9. Secoli SR, Figueras A, Lebrao ML, de Lima FD, Santos JLF (2010) Risk of potential drug-drug interactions among Brazilian elderly. Drug Aging 27: 759770 .

10. Hines LE, Malone DC, Murphy JE (2012) Recommendations for generating evaluating, and implementing drug-drug interaction evidence. Pharmacother $J$ Human Pharmacol Drug Ther 32: 304-313.

11. Yu J, Zhou Z, Tay-Sontheimer J, Levy RH, Ragueneau-Majlessi I (2018) Risk of clinically relevant pharmacokinetic-based drug-drug interactions with drugs approved by the US Food and drug administration between 2013 and 2016 . Drug Metab Disposition 117.

12. Greenblatt DJ (2017) Mechanisms and consequences of drug-drug interactions. Clin Pharmacol Drug Dev 6: 118-124.

13. Ramos-Esquivel A, Víquez-Jaikel Á, Fernández C (2017) Potential drug-drug and herb-drug interactions in patients with Cancer: a prospective study of medication surveillance. J Oncol Prac 13: e613-e622.

14. Van Leeuwen RWF, Brundel DHS, Neef C, van Gelder T, Mathijssen RHJ, et al. (2013) Prevalence of potential drug-drug interactions in cancer patients treated with oral anticancer drugs. British J Cancer 108: 1071-1078.

15. Gagne JJ, Maio V, Rabinowitz C (2008) Prevalence and predictors of potential drug-drug interactions in Regione Emilia-Romagna, Italy. J Clin Pharm Ther 33: 141-151.

16. Secoli SR, Figueras A, Lebrao ML, de Lima FD, Santos JLF (2010) Risk of potential drug-drug interactions among Brazilian elderly. Drug Aging 27: 759770.

17. Ismail M, Iqbal Z, Khattak MB, Javaid A, Khan TM (2011) Prevalence, types and predictors of potential drug-drug interactions in pulmonology ward of a tertiary care hospital. African J Pharm Pharmacol 5: 1303-1309. 
Citation: Hamoudi NM (2018) Does Drug-drug Interactions Attain Enough Attention from Health Care Providers?. Pharm Anal Acta 9: 598. doi: $10.4172 / 2153-2435.1000598$

Page 4 of 4

18. Riechelmann RP, Zimmermann C, Chin SN, Wang L, O'Carroll A, et al. (2008) Potential drug interactions in cancer patients receiving supportive care exclusively. J Pain Symp Manage 35: 535-543.

19. Passarelli MCG, Jacob-Filho W, Figueras A (2005) Adverse drug reactions in an elderly hospitalised population. Drug Aging 22: 767-777.

20. Uijtendaal EV, van Harssel LL, Hugenholtz GW, Kuck EM, Zwart-van Rijkom $\mathrm{JE}$, et al. (2014) Analysis of potential drug-drug interactions in medical intensive care unit patients. Pharmacother J Human Pharmacol Drug Ther 34: 213-219.

21. Ismail M, Iqbal Z, Khan MI, Javaid A, Arsalan H, et al. (2013) Frequency, levels and predictors of potential drug-drug interactions in a pediatric ward of a teaching hospital in Pakistan. Trop J Pharm Res 12: 401-406.

22. Ismail M, lqbal Z, Khattak MB, Khan MI, Javaid A, et al. (2012) Potential drugdrug interactions in cardiology ward of a teaching hospital. Health Med 6: 16181624.

23. Faulx MD, Francis GS (2008) Adverse drug reactions in patients with cardiovascular disease. Curr Prob Cardiol 33: 703-768.

24. Murtaza G, Khan MYG, Azhar S, Khan SA, Khan TM (2016) Assessment of potential drug-drug interactions and its associated factors in the hospitalized cardiac patients. Saudi Pharm J 24: 220-225.

25. Subramanian A, Adhimoolam M, Kannan S (2018) Study of drug-drug interactions among the hypertensive patients in a tertiary care teaching hospital. Pers Clin Res 9: 9-14.

26. Chelkeba L, Alemseged F, Bedada W (2017) Assessment of potential drugdrug interactions among outpatients receiving cardiovascular medications at Jimma University specialized hospital, South West Ethiopia. Int J Basic Clin Pharmacol 2: 144-152.

27. Juhlin T, Björkman S, Höglund P (2005) Cyclooxygenase inhibition causes marked impairment of renal function in elderly subjects treated with diuretics and ACE $\square$ inhibitors. Eur J Heart Failure 7: 1049-1056.

28. Andersson ML, Böttiger Y, Lindh JD, Wettermark B, Eiermann B (2013) Impact of the drug-drug interaction database SFINX on prevalence of potentially serious drug-drug interactions in primary health care. Eur J Clin Pharmaco 69: $565-571$
29. Tannenbaum C, Sheehan NL (2014) Understanding and preventing drug-drug and drug-gene interactions. Expert Rev Clin Pharmacol 7: 533-544.

30. Riechelmann R, Girardi D (2016) Drug interactions in cancer patients: A hidden risk?. J Res Pharm Prac 5: 77-78.

31. Scripture CD, Figg WD (2006) Drug interactions in cancer therapy. Nature Rev Canc 6: 546.

32. Tatro DS (2000) Drug interaction facts: 2001 Edition. Facts and Comparisons.

33. Hansten PD, Horn JR (2000) Drug interactions: Analysis and management: A clinical perspective and analysis of current developments. Facts and Comparisons.

34. Queneau P, Bannwarth B, Carpentier F, Guliana JM, Bouget J, et al. (2007) Emergency department visits caused by adverse drug events. Drug Saf 30: $81-88$.

35. Kheshti R, Aalipour M, Namazi S (2016)A comparison of five common drug-drug interaction software programs regarding accuracy and comprehensiveness. J Res Pharm Prac 5: 257.

36. Glassman PA, Simon B, Belperio P, Lanto A (2002) Improving recognition of drug interactions: Benefits and barriers to using automated drug alerts. Med Care 40: 1161-1171.

37. Ko Y, Malone DC, Skrepnek GH, Armstrong EP, Murphy JE, et al. (2008) Prescribers' knowledge of and sources of information for potential drug-drug interactions. Drug Saf 31: 525-536.

38. Kim BY, Sharafoddini A, Tran N, Wen EY, Lee J (2018) Consumer mobile apps for potential drug-drug interaction check: systematic review and content analysis using the Mobile App Rating Scale (MARS). JMIR mHealth uHealth 6: e74.

39. Nightingale G, Pizzi LT, Barlow A, Barlow B, Jacisin T, et al. (2018) The prevalence of major drug-drug interactions in older adults with cancer and the role of clinical decision support software. J Geriatr Oncol 9: 526-533.

40. Humphrey K, Jorina M, Harper M, Dodson B, Kim SY, et al. (2018) An investigation of drug-drug interaction alert overrides at a pediatric hospital. 8: 293-299.

41. Makiani MJ, Nasiripour S, Hosseini M, Mahbubi A (2017) Drug-drug interactions: The importance of medication reconciliation. J Res Pharm Prac 6: 61. 